Historic, Archive Document

Do not assume content reflects current scientific knowledge, policies, or practices. 



\section{GIG HARBOR,}

\section{WHOLESALE DAHLIA LIST}

Prices are for three or more of one variety.

Guarantee. We guarantee our tubers to be true to name with a good live eye, and cre carefully packed to reach you in satisfactory condition.

Terms: Net cash before shipment. 25\% deposit will hold orders until April 1 st. Postage paid on orders amounting to ten dollars. Please include postage on small orders.

A. D. Livoni (S) Amun $\mathrm{Ra}(\mathrm{D})$ (-) Adah Pomroy (C) Ballet Girl (H.C.) Bessie Boston (S) -........ Bob Pleuse (H.C.) -......Black Diamond (S) _. Bizarre (C) Britisher (C) Clifton (Sing) Carmencita (D) Champagne (D) Cigarette (H.C.) -........City of Portland (P) -.... Copper $\mathrm{Xing}(\mathrm{S})$ Dady Butler (H.C.) -....-Dakota (D) Delice (D) Dreers Thite (S) Dreers Yellow (S) Earl Williams (D) Fiery Gem (Sing.) Flame of Fire (D) Gen Miles (S) (S) Gold Mledal (S) Golden Opportunity (S) -.--Golden North (D) Golden West (H.S.) -..... Garden Glow (P) -...... Insulinde (D) ........ Jersey's Beauty (D) -...-.-Jane Selby (D) Johnkeer Boreel (D) -.....John L. Childs (D) ......... J. H. Goodrich (D) -....... Kalif (H.C.) Kitty Dunlap (D) King of Shows (S) -

Bobbie -himee -...--.--.-.-.-.-....Belle of Springfield -.....Clara Harsh -...-.-.-. Dr. Jim Girlie Glow George Ireland Hazel -- - - Jessica J. J. Klymn Lassie
Price $.10 \mathrm{each}$ .25 .40 .25

.15

.35

.15

.20

.15

.35

.30

.25

.25

.20

.10

.30

.15

.15

.15

.20

.40

.20

.75

.20

.15

.20

.60

.15

.50

.40

.40

.15

.20

.20

.15

.20

.40

.40
Le Grande Manitou (D) -....... .15 each. Lake Pende Orielle (H.C.) -..- 2.50 Maude Adams (S) -........... .20 Marcella Gill (D) - -...... .15 Mars. Bouchon (C) -........ .15 Marmion (D) -

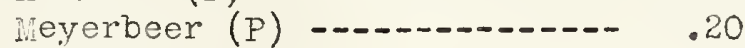
Millionaire (D) -......... .20 Mina Burgle (D) -.......... .10 Mrs. C. Sulback (D) -...-. .15 Mrs. Edna Spencer (C) -.--.-. .15 Mrs. E. F. T. Smith (P) - - . .25 Mrs. Estes (D) -... .25 Mrs. George Sheffield (D) -- . .60 Mrs. I. De Ver Warner (D) -.. .20 New Model (C) -........... .20 New Moon (C) _.................. . IFobilis (D) - . 45 Niebleungenhort (H.C.) - - . . . 20 Osum Shudor (D) -.......-. .25 Otargic (D) Pierrott (C) -........ .20 Paul Michael (D) -...-...-. .35 Patrich Omaro (D) - ........ .25 Polar Star (H.D.) -........ . .15 Purity (D) Pride of Calif. (D) -....... .15 Princess Pat (D) - ......... . .35 Pierre La Bland (C) -....-.. .10 Fosa Nell (D) - . 20 Snowdrift (D) -........... .20 Sweethearts Bouquet (P) -... .20 Tormy Atkins (D) - . . 35

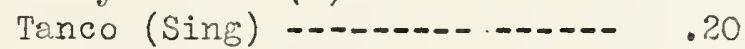
Tryphinnie (D) - .25 White Star (Sine.) -...... . . 20 Yellow King (H.C.) -...-... . 15

POM-PON DAHLIAS

.175 Little Jenny - . 15

.20 Little Lloyd -............ . 15

.15 Little Beeswing -.......... .15

.15 Little Bugler -.-.-.-.-.-.-. 15

.15 Nora Reynolds -.............. .15

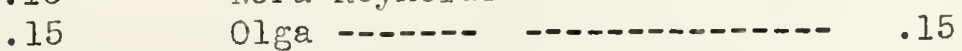

.20 Phoebe.--15

.20 San Toy -.-.-.-.-. 15

.20 Snow-clad - 10

.15 Tom Thumb - $.0 . . .-20$

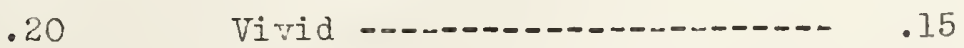

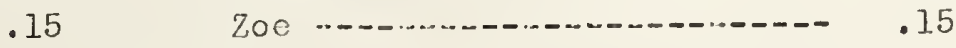


